\title{
Reinforced conservative management of post-dural puncture headache in a patient with a rare case of tethered cord syndrome using an abdominal binder: a case report
}

\author{
Seong-Ho Ok ${ }^{1}$, Miyeong Park ${ }^{1}$, Hokyung $\mathrm{Yu}^{1}$, Jiyoung Park ${ }^{1}$, Ju-Tae Sohn², Sunmin Kim ${ }^{3}$, \\ Kyeong Eon Park ${ }^{3}$, and Yeran Hwang ${ }^{3}$ \\ ${ }^{1}$ Gyeongsang National University Changwon Hospital \\ ${ }^{2}$ Gyeongsang National University College of Medicine \\ ${ }^{3}$ Gyeongsang National University Hospital
}

November 20, 2020

\begin{abstract}
Careful physical examination of the site of procedure before the caudal pain procedure should be performed because it could show the patient's abnormal anatomical conditions. An abdominal binder could be used effectively in a patient showing CSF leakage in the coccygeal area, which is not controlled by conventional compressive dressing.
\end{abstract}

\section{Introduction}

In a study conducted in Korea, it was found that only $0.8 \%$ of 2669 participants had an anatomical variation wherein the dural sac extended below S3, while 1.6\% had pathological conditions like perineural cysts and meningoceles. ${ }^{[1]}$ The incidence of post-dural puncture headache (PDPH) after caudal epidural block due to these anatomical abnormalities was low and its occurrence difficult to predict. ${ }^{[2]}$ Epidural blood patch (EBP), considered a gold standard treatment in PDPH, can lead to improvement of symptoms in more than $90 \%$ of cases. ${ }^{[3]}$ However, EBP cannot be performed on PDPH patients who do not respond to conservative management due to possible complications.

We report our experience in diagnosing rare tethered cord syndrome (TCS), which could cause PDPH, secondary to CSF leakage through an MRI. The MRI was performed to investigate the reason for subcutaneous cerebrospinal fluid (CSF) collection suspected by physical examinations and bedside sonography. PDPH accompanied by TCS was successfully resolved after using an abdominal binder, as reinforced conservative management, for a week.

\section{Case report}

A 59-year-old female visited our hospital due to posterior head and neck pain that developed after a coccygeal procedure. She was referred to the pain clinic for EBP.

She underwent the caudal approach procedure a week ago at a local clinic due to pain in the left leg and foot. Reports about the procedure performed from the clinic were inaccessible, but following the patient's description, a caudal epidural block was presumed to have been used. One day after the procedure, she found clear fluid leakage from the coccygeal area and started experiencing posterior headache. She received a disinfection and compressive dressing on the coccygeal area and was prescribed bed rest and pain killers from the local clinic, but her symptoms did not improve within a week. 
The numerical rating scale (ranging from 0 to 10, with a score of 0 representing no pain and 10 corresponding to intractable pain) score for pain severity was 5 and the pain was frequent, throbbing, and worsened by the orthostatic position.

On physical examination, the compressive dressing looked clean except for some swelling around the sacral area, and no leakage was observed. Bedside ultrasound (US) showed subcutaneous edema and fluid collection in the swollen peri-coccygeal area (Fig1). There were no suspicious signs of meningeal irritation such as fever or neck stiffness. Laboratory examination revealed no leukocytosis and normal C-reactive protein levels.

We conducted the following treatments under suspicion of PDPH with subarachnoid-cutaneous fistula after the caudal procedure.

First, she was admitted for absolute bed rest, hydration with intravenous fluid, and was prescribed acetaminophen as a painkiller. A neurologist referred her to a pain clinic for EBP because her headache lasted for more than a week despite conventional conservative management from a local clinic. We initially did not consider EBP through the sacral hiatus as a treatment plan, because there was a possibility of infection in the presence of a fistula or repeated CSF leakage. MRI was scheduled for checking the presence of subcutaneous CSF leakage and its cause. Additionally, we decided to disinfect the wound and apply compressive dressing using an obliquely applied abdominal binder around the patient's buttocks and the lower abdomen for better compact compression than that with the previous conventional compressive dressing, which comprised of adhesive tape and gauze.

After admission, a radiologist reported MRI, showed a low-lying conus medullaris (which reached down to the inferior border of the S2 vertebra, Fig 2) with a thickened filum terminale, an $8 \times 7 \mathrm{~cm}^{2}$ sized fatty contiguous mass with a placode at the sacrococcygeal area, and interspersed fluid around the mass. (Fig $3 \mathrm{~A}$ and $\mathrm{B}$ ) This showed that she had a rare TCS that was now discovered late in adulthood. A surgical procedure should have been considered for TCS, but since she did not have any symptoms of the cauda equina syndrome or any other severe neurologic symptoms, except the initial symptoms of left leg and foot pain, we decided to use reinforced conservative management with an abdominal binder first for a week.

On the 3rd day of hospitalization, the headache and neck pain decreased, but reappeared after a short walk. On the 4th day, she could walk without headache and neck pain, but she could still sometimes experience mild posterior neck pain. On the 5th day, US still showed irregular and mild hypoechoic subcutaneous edema around the coccyx area and suspicious fluid collection, but its size had decreased. On the 6th day, she was discharged with an abdominal binder.

One week after discharge, she visited the pain clinic and had no complaints even without the abdominal binder.

\section{Discussion}

TCS presents various symptoms depending on age and its cause. Neurologic symptoms, sensory or motor dysfunction, dysuria, and deformation of the feet or spine are usually developed by progressive stretching of and increased tension in the spinal cord in children. ${ }^{[4]}$ Cutaneous stigmata, hair tufts, skin tags, dimple, nevi, hemangioma, lumps, lipomas, etc. could be surface (cutaneous) findings that suspect TCS. ${ }^{[4]}$ Our patient had subcutaneous fluid collection following the caudal approach and it looked like it was concealing the existence of a subcutaneous lipoma. If a physician at a local clinic had performed a physical examination of the procedure site before the practice, he would have found a lesion suspected as a lump or lipoma. We suspected that she had a CSF leakage based on physical examination and bedside US and finally confirmed a rare case of TCS and subarachnoid-cutaneous fistula by MRI. Based on our experience, it is difficult to predict dural puncture before caudal epidural block without expensive imaging studies such as CT or MRI.

In children, an inelastic filum is attached to the caudal end of the spinal cord which adds continuous tension to the spinal cord causing severe neurological symptoms. Surgical treatment of TCS should maintain neural functions and circulation of CSF, and reconstruct the dural sac; additionally, there should be no adverse effects, such as infection or adhesion. ${ }^{[5]}$ Major symptoms of TCS, such as pain, bowel or urinary dysfunction, 
may not improve after surgery in adults, and postoperative complications of old age may be three times higher than that in young patients. Asymptomatic adult TCS patients could be followed up only for symptoms, and conservative management could be applied to non-aggressive and bearable symptoms. ${ }^{[6-8]}$ There is a controversy over the superiority of conservative management and surgical management. This case showed that conservative management could improve symptoms in adult TCS patients, therefore, we should be careful in our decision about employing surgical treatment. ${ }^{[6-8]}$ In our patient, surgical release of the filum terminale anchored to the spinal cord could be considered, but we decided to maintain the conservative management with an abdominal binder and observation of the symptoms because her mild neurologic symptoms (pain of the left leg and foot) improved and PDPH decreased.

In the structures of the epidural cavity, the incomplete lateral wall could cause peritoneal pressure to be transmitted to the epidural cavity through the intervertebral foramina. Therefore, increased abdominal pressure could induce an increase in the epidural pressure. ${ }^{[9]}$ In this respect, the compression of the abdomen with an abdominal binder would be helpful to decrease the PDPH after spinal tapping in postpartum patients by increasing the peritoneal pressure. The preventive effects of an abdominal binder in PDPH's occurrence were similar to that of the injection of normal saline into the epidural space. ${ }^{[10]}$ We applied an abdominal binder obliquely for two main purposes. First, we tried to maintain high epidural pressure by increasing peritoneal pressure to reduce pressure variations due to change in posture. Second, compressive dressing with gauze and adhesive tape was insufficient, especially in the coccyx area because the movable and soft buttocks would offset the adhesive tape's compression. Considering these points, we applied an abdominal binder to surround the lower abdomen, buttocks, and coccyx, obliquely from the upper anterior pelvis to the lower posterior pelvis. This placement, fastening the binder around the pelvis, prohibited lateral movement of the buttocks and provided evenly distributed compression of the lipoma and large subcutaneous CSF collection. The abdominal binder was able to compress a surface area of $8 \times 7 \mathrm{~cm}^{2}$.

The EBP was a very effective treatment for spontaneous intracranial hypotension; therefore, $90 \%$ of the patients showed improvement regardless of the presence or location of CSF leakage. ${ }^{[11]}$ However, complications and/or side effects - ranging from mild to severe - make EBP a challenging procedure for anesthesiologists. Mild events include temporary nerve compression syndrome, dizziness, vertigo, or tinnitus, while the rare, severe adverse effects include epidural abscess or cord compression. ${ }^{[12]}$ Our patient had a history of body fluid leakage, which could be expected as the puncture site had swelling and fluctuation on physical examination of the coccygeal area, which appeared as a subcutaneous fluid collection. Because we confirmed that this fluid collection communicated with the intrathecal space via MRI (Fig 3A and B), we thought that the EBP through the sacral hiatus had a high risk of meningitis. It was reported that the large volume of blood infused into the epidural space ran in the cephalad direction during EBP in MRI, ${ }^{[13]}$ and thus, the lumbar site for EBP in our patients seemed ineffective and large volumes of blood would be needed in contrast to that in the sacral approach if it was performed.

Based on the findings of this case, the following points should be kept in mind during the caudal procedure.

1. Careful physical examination of the procedure site before the caudal pain procedure should be performed because it could show the patient's abnormal anatomical conditions.

2. When a subcutaneous fluid collection after a neuraxial procedure is suspected by peripheral swelling or fluctuation, fluid collection can be examined by easily accessible US. If it has been proven, CSF leakage must be ruled out by an MRI scan, and then EBP should be considered later.

3. An abdominal binder could be used effectively in a patient showing CSF leakage in the coccygeal area, which is not controlled by conventional compressive dressing.

Acknowledgments: We would like to thank Editage (www.editage.co.kr) for English language editing.

Disclosures: The authors have read the CARE Checklist (2013), and the manuscript was prepared and revised according to the CARE Checklist (2013).

Ethical concerns: The study was approved by the institutional review board of Gyeongsang National University Changwon Hospital (GNUCH 2020-08-018) 
Conflict-of-interest: The authors declare that they have no conflict of interest.

\section{Author contributions :}

Miyeong Park : drafting the manuscript and primary treatment provider

Kyeong Eon Park, Yeran Hwang : drafting the manuscript

Hokyung Yu, Jiyoung Park, Sunmin Kim : co-authors responsible for the literature review

Ju-Tae Sohn, Seong-Ho Ok : the senior authors managing the construction and edits of the manuscript and guiding through the submission process.

\section{References:}

1. Joo J, Kim J, Lee J. The prevalence of anatomical variations that can cause inadvertent dural puncture when performing caudal block in Koreans: a study using magnetic resonance imaging. Anaesthesia.2010;65(1):23-26.

2. Kim SG, Yang JY, Kim DW, Lee YJ. Inadvertent Dural Puncture during Caudal Approach by the Introducer Needle for Epidural Adhesiolysis Caused by Anatomical Variation. The Korean journal of pain.2013;26(2):203-206.

3. So Y, Park JM, Lee PM, Kim CL, Lee C, Kim JH. Epidural Blood Patch for the Treatment of Spontaneous and Iatrogenic Orthostatic Headache.Pain Physician. 2016;19(8):E1115-e1122.

4. Hertzler DA, DePowell JJ, Stevenson CB, Mangano FT. Tethered cord syndrome: a review of the literature from embryology to adult presentation. Neurosurgical focus. 2010;29(1):E1.

5. Kang JK, Yoon KJ, Ha SS, Lee IW, Jeun SS, Kang SG. Surgical management and outcome of tethered cord syndrome in school-aged children, adolescents, and young adults. J Korean Neurosurg Soc.2009;46(5):468471.

6. Duz B, Gocmen S, Secer HI, Basal S, Gonul E. Tethered cord syndrome in adulthood. J Spinal Cord Med. 2008;31(3):272-278.

7. Tu A, Steinbok P. Occult tethered cord syndrome: a review.Child's Nervous System. 2013;29(9):16351640 .

8. Drake JM. Occult tethered cord syndrome: not an indication for surgery. 2006;104(5):305-308.

9. Hogan QH. Epidural anatomy: new observations. Canadian Journal of Anaesthesia. 1998;45(S1):R40R48.

10. Mosavy SH, Shafei M. Prevention of headache consequent upon dural puncture in obstetric patient. Anaesthesia. 1975;30(6):807-809.

11. Smith KA. Spontaneous intracranial hypotension: Targeted or blind blood patch. J Clin Neurosci. 2016;25:10-12.

12. White B, Lopez V, Chason D, Scott D, Stehel E, Moore W. The lumbar epidural blood patch: A Primer. Applied Radiology.2019;48(2):25-30.

13. Beards S, Jackson A, Griffiths A, Horsman E. Magnetic resonance imaging of extradural blood patches: appearances from 30 min to 18 h.BJA: British Journal of Anaesthesia. 1993;71(2):182-188.

\section{Figure legends}

Figure 1. Sonographic longitudinal view of the coccygeal area, showing the subcutaneous edema and fluid collection (orange arrow) in the peri-coccygeal area. 
Figure 2. The end of sunken conus medullaris (orange arrow) extending to the S2 vertebra's inferior border with the thickened filum terminale in the sagittal T2 weighted magnetic resonance imaging (MRI).

Figure 3. An approximately $8 \times 7 \mathrm{~cm}^{2}$-sized fatty contiguous mass with neural placode at the sacrococcygeal area and the interspersed fluid around the coccygeal mass as seen on horizontal (A) and sagittal (B) sections on T1-weighted magnetic resonance imaging (MRI).

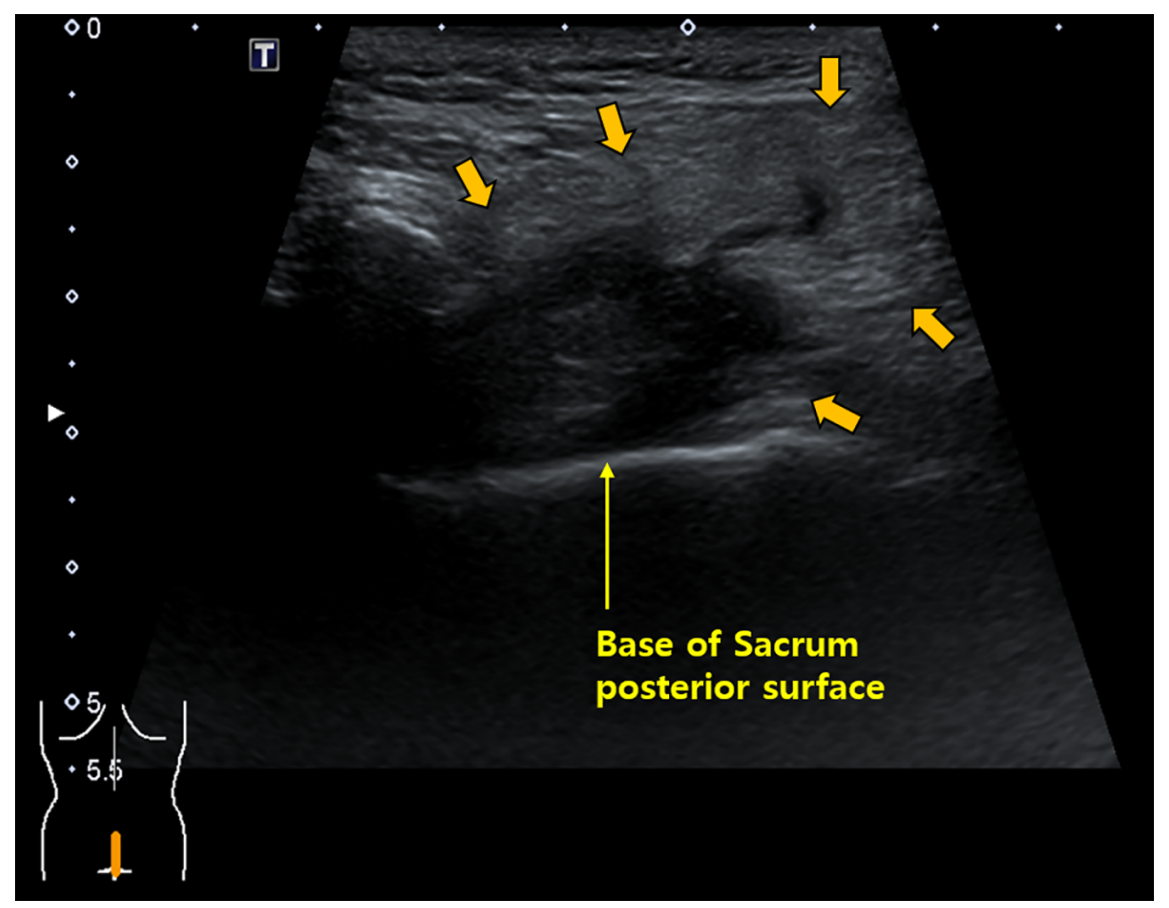




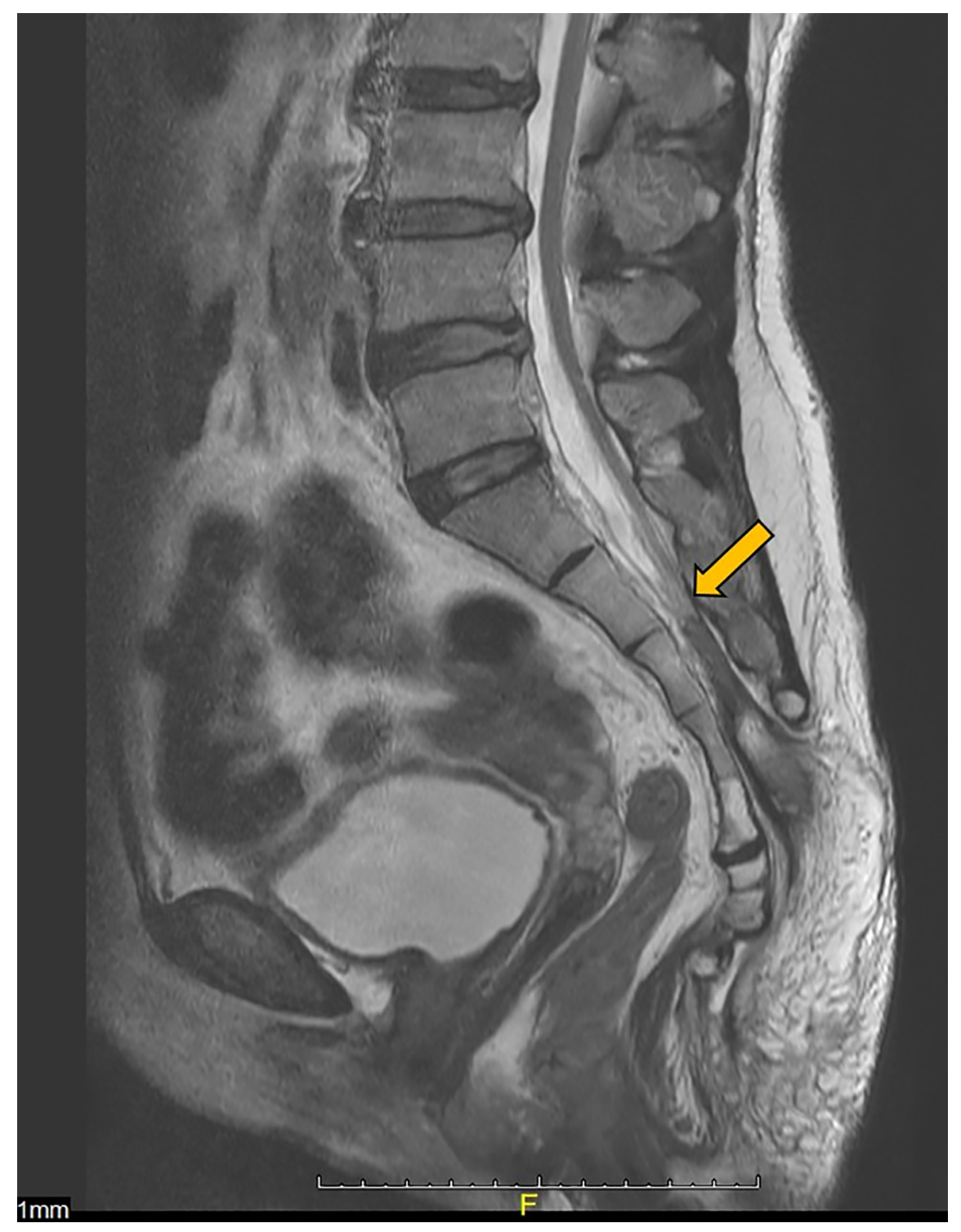



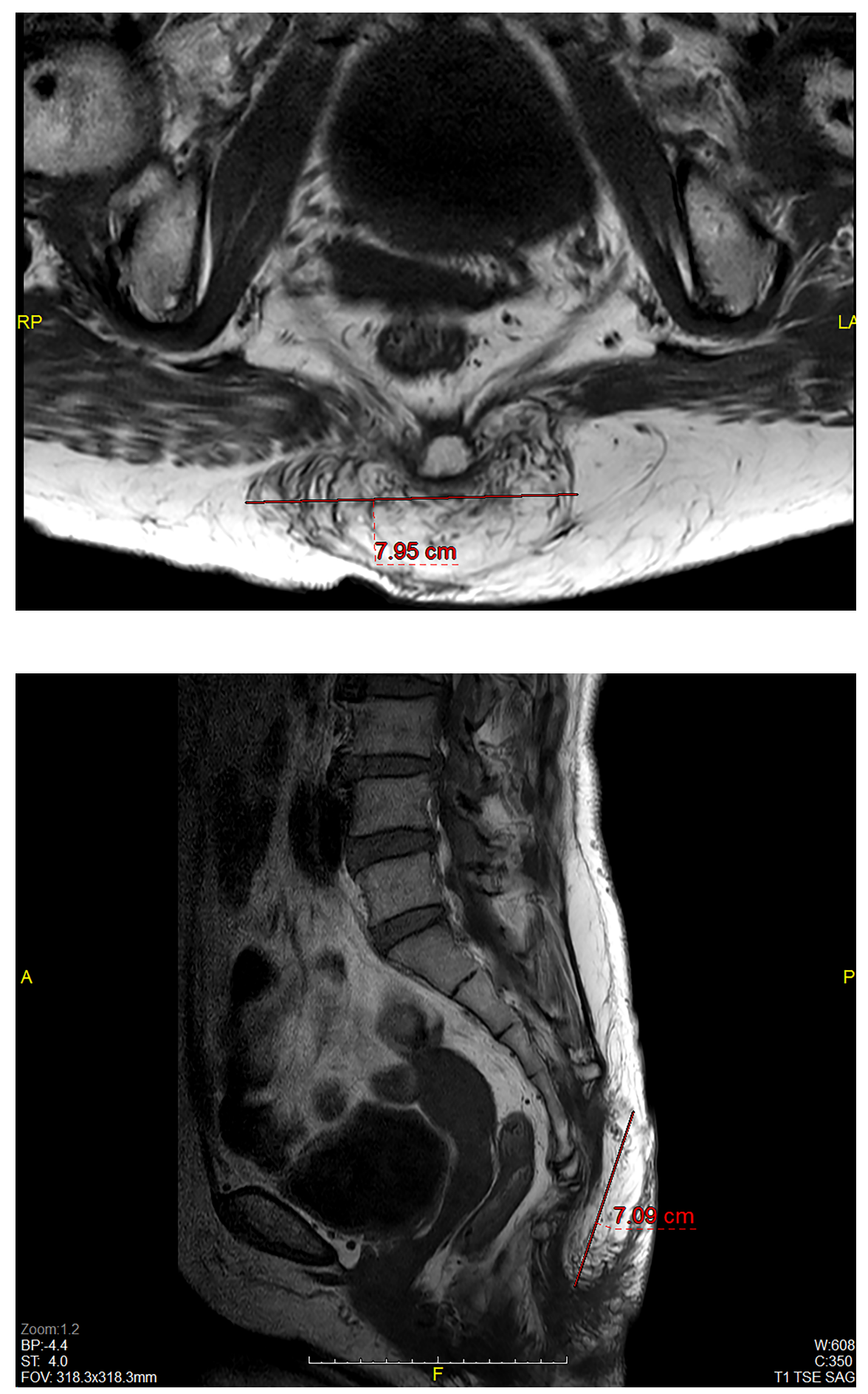